\title{
A POOLED ANALYSIS OF CONCURRENT CHEMORADIOTHERAPY (CCRT) FOR PATIENTS WITH STAGE III NON-SMALL CELL LUNG CANCER (NSCLC) WHO PARTICIPATED IN U.S. COOPERATIVE GROUP TRIALS: COMPARING THE OUTCOMES OF ELDERLY TO YOUNGER PATIENTS (PTS)
}

BACKGROUND: CCRT is the standard treatment (TRT) for stage 3 NSCLC. Elderly pts are common and may have increased toxicity and poorer results from CCRT.

METHODS: We collected individual patient data (IPD) of pts who participated in cooperative group phase 2/3 trials of CCRT for stage 3A/3B pts from 1990-2012. We compared the overall survival (OS), progression-free survival (PFS), and adverse events (AE's) for pts age $\geq 70$ years (yrs) (elderly) vs. $<70$ yrs (younger). Unadjusted and adjusted Hazard Ratios (HRs) for survival time and their confidence intervals (CIs) were estimated by single-predictor and multivariable Cox models. Unadjusted and adjusted Odds Ratios (ORs) for AE's and their CIs were obtained from single-predictor and multivariable logistic regression models. RESULTS: IPD from 15 trials were analyzed; 2243 pts were younger and 702 were elderly. Median OS and PFS for elderly and younger pts are in the table. In the unadjusted and multivariable models elderly pts had worse OS $(\mathrm{HR}=1.19 ; 95 \% \mathrm{CI}=1.08-1.31$, and 1.18; 95\% CI=1.06-1.32, respectively). In the unadjusted \& multivariable models, elderly and younger pts had a similar PFS $(\mathrm{HR}=1.05 ; 95 \% \mathrm{CI}=0.96-1.15$ and $1.06,95 \% \mathrm{CI}=0.95-1.17$, respectively $)$. The rates of AE's and TRT related deaths are presented in the table. Elderly pts had a higher rate of AE's in the unadjusted \& multivariable models $(\mathrm{OR}=1.27 ; 95 \% \mathrm{CI}=0.99-1.63$ and 1.33; 95\% CI=1.04-1.72, respectively). A lower percentage of elderly pts completed TRT (50.9\% and $61.2 \%$, respectively; $\mathrm{P}<0.0001)$ and higher percentage stopped due to AE's $(20.5 \%$ and $14.1 \% ; P<0.0001)$. CONCLUSIONS: Elderly pts enrolled in cooperative group CCRT trials had worse OS, but similar PFS. The rates of severe AE's was higher and a lower percentage of elderly pts completed TRT.

\begin{tabular}{|lccc|}
\hline & Elderly & Younger & P-value $^{\text {a }}$ \\
\hline Median OS (months) & 15.3 & 17.7 & 0.0002 \\
Median PFS (months) & 8.6 & 9.1 & 0.2858 \\
All toxicities grade $\geq \mathbf{3}$ & $87 \%$ & $84 \%$ & 0.0575 \\
Hematologic AE's grade $\geq \mathbf{3}$ & $65 \%$ & $61 \%$ & 0.0804 \\
Non-hematologic AE's $\geq \mathbf{3}$ & $67 \%$ & $59 \%$ & 0.0002 \\
Grade 5 AE's & $7.8 \%$ & $3.9 \%$ & $<0.0001$ \\
TRT related deaths & $3.2 \%$ & $2.0 \%$ & 0.1233 \\
\hline
\end{tabular}

${ }^{a}$ Log-rank test for survival times, chi-square test for AE's, and Fischer exact test for TRT related deaths. 